\title{
Solid-State Technologies for Flexible and Efficient Marine DC Microgrids
}

S. Kim, J. Kucka, G. Ulissi, et al.

This material is posted here with permission of the IEEE. Such permission of the IEEE does not in any way imply IEEE endorsement of any of EPFL's products or services. Internal or personal use of this material is permitted. However, permission to reprint / republish this material for advertising or promotional purposes or for creating new collective works for resale or redistribution must be obtained from the IEEE by writing to pubs-permissions@ieee. org. By choosing to view this document, you agree to all provisions of the copyright laws protecting it. 


\title{
Solid-State Technologies for Flexible and Efficient Marine DC Microgrids
}

\author{
Seongil Kim, Member, IEEE, Jakub Kucka, Member, IEEE, Gabriele Ulissi, Graduate Student Member, IEEE, \\ Soo-Nam Kim, and Drazen Dujic, Senior Member, IEEE
}

\begin{abstract}
This paper presents flexible operating modes of marine DC microgrids and main advantages of employing energy storage systems into such DC microgrids. Scaled-down marine systems with two DC buses and integrated energy storages are considered in this paper. To verify superior features of the DC microgrids with solid-state technologies and energy storage systems, a wide range of system operating modes are experimentally demonstrated, ranging from basic to advanced operating modes: DC voltage regulation, power sharing control, soft start (smoothly charging bus potential to the nominal level), seamless transition (transiting open-bus and closed-bus operations), load leveling (flattening generator loads by energy storage systems), transient mitigation (mitigating sudden load changes by energy storage systems), and zero-emission (powering the marine networks by energy storage systems near a port). The experimental results show that DC microgrids with solid-state technologies and energy storage systems provide ship owners with the flexibility in energy management of the system, fuel savings, and environmental compliance in a port.
\end{abstract}

Index Terms-DC Microgrids, Energy Storage Systems, Marine Power Distribution Networks, Solid-State Technologies.

\section{INTRODUCTION}

The increasing penetration of renewable energy sources, energy storage systems (ESSs), and DC loads (e.g., data center and electric vehicles) has opened the door to DC technology to pave its way into future grids. The shift has been more obvious in the marine domain supported by track records of installed onboard DC solutions since 2013 [1], [2].

A ship is an off-grid stand-alone system (a microgrid) without the need to comply with strict utility grid codes, which allows for the greater flexibility to utilize new concepts in design of power system. This is one of the reasons why the movement toward DC has been proceeding actively in the marine sector. In addition to novel technologies, DC solutions are bringing directly quantifiable advantages, such as fuel savings with variable-speed generators, easy integration of ESSs, weight and footprint reduction in electrical installations, and optimization of running engines [3]-[6].

Manuscript received August 21, 2020; revised December 29, 2020; accepted March 5, 2021. (Corresponding author: Seongil Kim). This work was supported by Hyundai Electric \& Energy Systems.

S. Kim and S.-N. Kim are with the Hyundai Electric \& Energy Systems Research Centre, Yongin 16891, Republic of Korea (e-mail: kim.seongil@hyundai-electric.com; kimsoonam@hyundai-electric.com).

J. Kucka, G. Ulissi, and D. Dujic are with the Power Electronics Laboratory of École Polytechnique Fédérale de Lausanne (EPFL), 1015 Lausanne, Switzerland (e-mail: jakub.kucka@epfl.ch; gabriele.ulissi@epfl.ch; drazen.dujic@epfl.ch).
A solid-state bus-tie switch (SSBTS) plays an important role in system protection of marine DC microgrids. The SSBTS is used for the bus separation in the range of several tens of microseconds [7]-[9]. It allows for promptly clearing DC faults and avoiding converter damages. This active device also offers advantages on the system level by two novel functions of the SSBTS at no additional cost, beside the protection function: soft start - smoothly charging bus potential to the nominal level; and seamless transition - promptly supplying electric power to the bus experienced a power outage during the open-bus operation. The soft start ability by the SSBTS provides softly energizing bus without additional components for the inrush current limiting, allowing space and cost savings. Moreover, the seamless transition can improve the system reliability by promptly taking power from the healthy side in the event of a generator failure during the open-bus operation.

In the past decade, there has been enormous evolution in ESSs, especially in Li-ion batteries, in terms of energy density, power density, and cost [10], [11]. This progress allows ESSs to be accepted more widely in marine applications [1], [12]. While an ESS is still a costly option, it provides several main benefits for ship owners, mostly related to fuel savings and environmental compliance in a port: transient mitigation - supporting transient demands by ESSs with short time constants to improve the transient performance of diode and thyristorbased DC microgrids; load leveling (peak shaving) - operating generators at optimal loads by storing energy during light load conditions, discharging it during too heavy load conditions, and reducing the number of running generators; and zeroemission - turning $O F F$ generators and powering the networks by ESSs when a ship is entering or exiting a port to avoid air pollutants from the ship. By avoiding transient generator loads, it is possible to increase the lifespan of a ship engine-generator set and reduce air pollutants generated during high transient accelerations and decelerations. The flattened generator loads by the load leveling provide a large potential for fuel savings in marine applications. There is a wide range of activities to make a cleaner port. The zero-emission operating mode is crucial to achieve the goal of becoming the zero-emission port.

This paper presents various performance improvements in marine DC microgrids, offered by above discussed technologies. Complete small-scale two-bus DC system is established, to support experimental investigations and evidence of effects of selected technologies on various operating modes. Section II describes the lab-scaled hardware test setup to replicate marine two-bus DC microgrids of $500 \mathrm{~V}$. The novel soft start and 


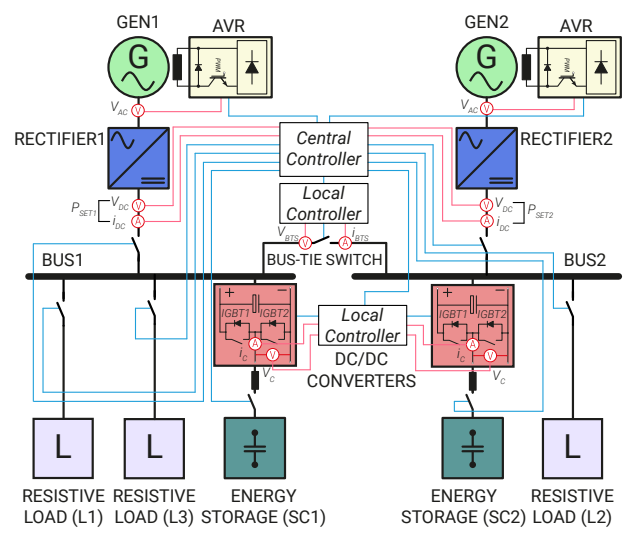

Fig. 1: Schematic diagram of marine DC microgrids.

seamless transition modes, which can make the DC microgrids more flexible and reliable, are demonstrated in Section III. In Section IV, practical ESS applications in marine DC networks are experimentally demonstrated, including control, utilization, and expected benefits of ESS. Furthermore, in the same section, the power droop-based zero-emission mode is newly demonstrated. The last section summarizes the main results.

\section{Hardware Test SETUP}

This section describes hardware components of the test setup. The test setup is composed of two DC motor-generator sets, two diode rectifier systems, a SSBTS, two supercapacitor banks, resistive loads, and a central controller (see Fig. 1). Each component is described hereafter. Note that, as the part of the supply system, the diode rectifier which shows the best stability performance among the possible three rectifier systems (diode, thyristor, and active rectifiers) is employed. Moreover, relatively short DC lines are rather consequence of laboratory infrastructure, resulting in rather low inductances in the system, to avoid system instability issues [13].

\section{A. DC Motor-Generator Sets and Rectifiers}

Thanks to the use of rectifiers to provide DC supply, generator design (frequency, number of poles, voltage level) can be optimized with much more degrees of freedom than in AC system. To demonstrate the flexibility in the selection of generator frequency, two generator sets are utilized in the test setup: GEN1 - $10 \mathrm{~kW}, 380 \mathrm{~V}$, operated at fixed $50 \mathrm{~Hz}$; and GEN2 - $25 \mathrm{~kW}, 380 \mathrm{~V}$, operated with variable frequency in the range of $200-400 \mathrm{~Hz}$.

Each DC motor-synchronous generator set includes a DC motor drive, a DC motor, a synchronous generator, and an automatic voltage regulator (AVR), as shown in Fig. 2a and 2b. The DC motor driven by a thyristor power converter emulates a prime mover in marine systems, i.e., diesel engine, typically found on a ship. The generator terminal voltage is regulated by a commercial AVR (ABB Unitrol 1005) that is an onequadrant AVR based on the DC chopper (a buck converter).

A three-phase six-pulse diode rectifier is employed to each generator set for the rectification as the diode rectifier is simpler, more robust, and more cost-effective than a thyristor

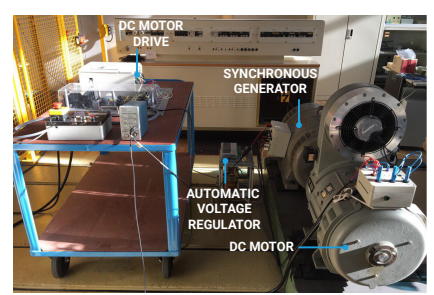

(a)

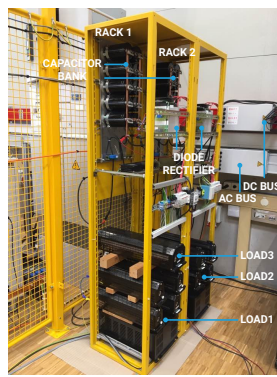

(c)

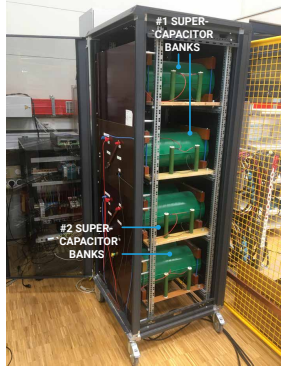

(d)

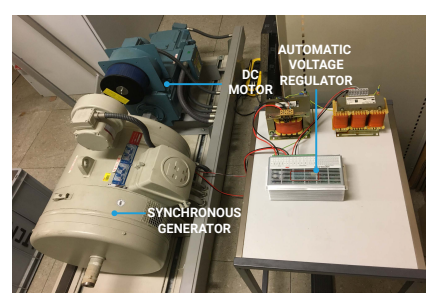

(b)

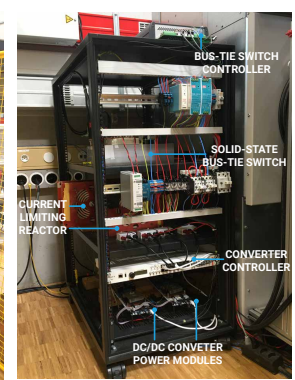

(e)
Fig. 2: Actual implementation: (a) GEN1, (b) GEN2, (c) rectifier systems, (d) supercapacitor banks, and (e) SSBTS and DC-DC converters.

rectifier and a voltage source converter that have also been applied for the marine power supply systems [14]. DC-link capacitor bank at rectifier output is rated for $500 \mathrm{~V}$ and is made modular allowing for capacitance to be adjusted from $2.3 \mathrm{mF}$ up to $11.5 \mathrm{mF}$. The rectifier systems assembled in the racks are presented in Fig. 2c.

\section{B. Solid-State Bus-Tie Switch}

As one of the key protection components, a SSBTS developed in the work of [15] is integrated into the test setup. The bus-tie switch has the following ratings:

- Nominal voltage: $600 \mathrm{~V}$

- Rated current: $50 \mathrm{~A}$

- Trip current: $100 \mathrm{~A}$

- Maximum allowed current: $200 \mathrm{~A}$

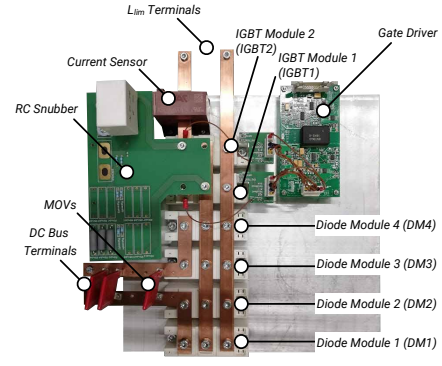

(a)

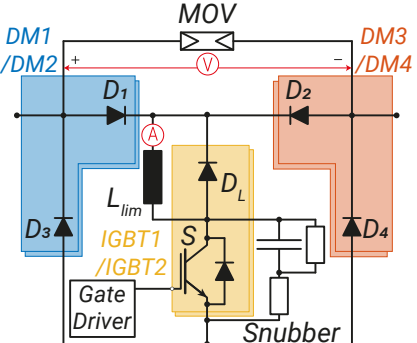

(b)
Fig. 3: SSBTS [15]: (a) actual implementation and (b) schematic diagram. A voltage sensor and a current limiting reactor are not presented in Fig. 3a as they are external components. 
The switch topology is based on a four-quadrant switch configuration [four diodes $\left(D_{1}, D_{2}, D_{3}\right.$, and $\left.D_{4}\right)$ and one IGBT module $(S)$ ] combined with a transient current limiting reactor $\left(L_{l i m}\right)$ and a freewheeling diode $\left(D_{L}\right)$ in series to the IGBT module, as shown in Fig. 3b. An RC snubber and metal oxide varistors (MOVs) are added to suppress transient overvoltages during the interruption.

The right-side supply forces the current to flow through $D_{2}$, $L_{l i m}, S$, and $D_{3}$ if the IGBT is the $O N$-state. For the left-side supply, the current direction is the opposite. The IGBT module is first turned $O F F$ in the event of a fault. This forces the overcurrent to flow through the snubber circuit and the MOVs. The energies stored in the current limiting reactor and the system stray inductance are dissipated by the diode of $D_{L}$ and the MOVs, respectively, until the interruption is terminated.

The assembled SSBTS is presented in Fig. 3a. On top of the heatsink, the mounted components are four diode modules, two IGBT modules, a gate driver, a PCB-based RC snubber circuit, MOVs, and a current sensor. Note that the current limiting reactor of $48 \mu \mathrm{H}$ and the voltage sensor are externally connected. To increase overcurrent withstand capability, two modules are installed in parallel for the left-side diodes $\left(D_{1}\right.$ $\left.D_{3}\right)$, the right-side diodes $\left(D_{2}-D_{4}\right)$, and the diode-IGBT modules $\left(D_{L}-S\right)$, as shown in Fig. 3b.

\section{Supercapacitor-Based Energy Storage Systems}

Due to lack of access to suitable batteries for the test setup, alternative technology (supercapacitor bank) is used, without major negative effect on the performances that this paper aims to demonstrate. Each supercapacitor bank is rated for $160 \mathrm{~kJ}$. These supercapacitor banks are coupled in the test setup via buck-boost topology-based DC-DC converters. Two half-bridge power modules are used for the converters and they are controlled by an Imperix B-Box controller. The central controller talks with the ESS controller through CANOpen communication. The actual implementation of the ESSs and the DC-DC converters is shown in Fig. 2d and Fig. 2e.

\section{Central Controller}

A central controller of the setup is implemented with Pixsys TD820 that is a human-machine interface (HMI) panel with an integrated programmable logic controller (PLC). Two analogue/digital input/output modules (Pixsys ETD1644) are installed at the backside of the PLC panel. The PLC communicates with the AVRs, the local controller of the SSBTS, and the local controller of the DC-DC converters. Analogue inputs and digital outputs of the PLC are used to acquire voltage and current information and to control the switches for the loads, respectively (see Fig. 1).

\section{Demonstration of Flexible Systems With SSBTS}

Various operating modes are demonstrated in the lab-scaled systems. The demonstrations are carried out for basic operating modes (voltage regulation and power sharing) and advanced operating modes (soft start and seamless transition), available with the novel functions in the SSBTS.

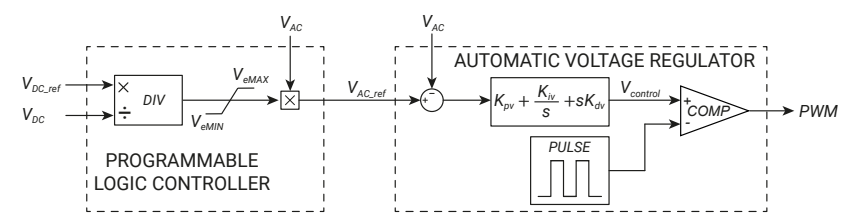

Fig. 4: Block diagram of DC voltage control.

\section{A. Voltage Regulation and Soft Start}

A DC voltage regulation is an important function of DC microgrid control to maintain a constant DC output voltage against system disturbances and load changes. In the test setup, the regulation function is realized with the combination of the PLC and the AVR. The utilized AVR is for AC networks, i.e., lack of the DC regulation function, and thus the PLC is combined to provide an $\mathrm{AC}$ reference corresponding to the DC voltage error, as shown in Fig. 4.

The PLC acquires the DC voltage information and calculates the voltage error. The new AC voltage reference is sent from the PLC to the AVR. The AVR finally regulates the AC generator terminal voltage with its PID controller. The parameters for the voltage regulation are provided in Table I.

In marine AC microgrids, synchronous generators have to be synchronized to the networks when they are interconnected. For the synchronization, four factors to be matched are phase sequence, voltage magnitude, frequency, and phase angle [16]. By contrast, the only factor to be considered is the voltage magnitude in marine DC microgrids. If the SSBTS can smoothly charge the potential from one bus to the other bus (called soft start in the work), there is no limitation on the voltage matching requirement during the interconnection process. Furthermore, it allows to extend operation sequences and/or modes of DC microgrids, e.g., supplying electric power to the bus in which no electric source is available due to system design, operation mode (one generator operation mode), or machine failure. The soft start function shown in Fig. 5 is performed by the controller of the SSBTS.

In addition to the roles of the SSBTS (bus connection and disconnection under normal conditions as well as fault interruption), the soft start ability requires only additional control functions for its implementation. The principle of the implemented soft start function is the switch being repeatedly turned $O N$ and $O F F$, and the connection of the two buses once the charging is complete. In detail, when the SOFT START ON command is transferred from the PLC, the pulse keeps the switch $O N$ for a short time. Due to the potential difference between the buses, the charging current $\left(i_{B T S}\right)$ flows via the switch, and then the switch is turned $O F F$ if the current reaches the threshold level or the pulse becomes $O F F$ with its duty cycle $(D)$. This action occurs at every pulse cycle and

TABLE I: Parameters for DC voltage regulation.

\begin{tabular}{lccccc}
\hline \hline Parameters & $V_{e M A X}$ & $V_{e M I N}$ & $K_{p v}$ & $K_{i v}$ & $K_{d v}$ \\
\hline GEN1 & 1.05 & 0.95 & 20 & 10 & 2 \\
GEN2 & 1.05 & 0.95 & 20 & 5 & 4 \\
\hline \hline
\end{tabular}




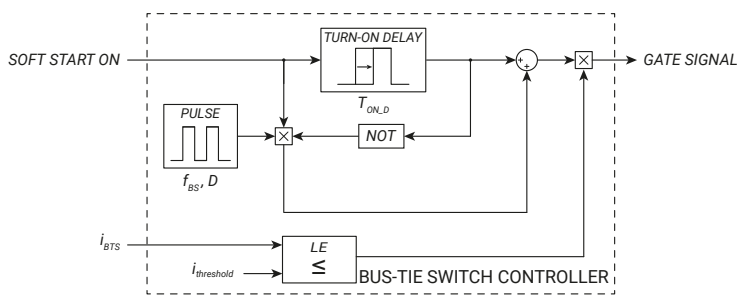

Fig. 5: Block diagram of soft start control. The used parameters for the soft start control are: $f_{B S}=100 \mathrm{~Hz}, D=0.1 \mathrm{pu}$, $T_{O N_{-} D}=5 \mathrm{~s}$, and $i_{\text {threshold }}=100 \mathrm{~A}$.

the potential of the bus being charged is gradually increased. Once the potential difference is within the acceptable range, the switch remains in the $O N$-state through the TURN-ON $D E L A Y$ block in Fig. 5. To verify the DC voltage regulation function and the soft start, two tests are conducted, as shown in Fig. 6 and Fig. 7.

In Fig. 6, BUS1 that is supplied by GEN1 is initially operated with its rated voltage. At $0 \mathrm{~s}$, the soft start command is issued and the SSBTS charges the potential of BUS2 by turning the SSBTS $O N$ at every $10 \mathrm{~ms}$ and $O F F$ once the charging current reaches the threshold value or the pulse becomes $O F F$ with its duty cycle, repetitively, as shown in Fig. $6 \mathrm{~b}$. The potential in BUS2 is fully charged at approximately $3.7 \mathrm{~s}$. Note that the transient current during the soft start is not measured in Fig. 6a due to the sampling rate limitation of a measuring device for a long period time $(10 \mathrm{~s} / \mathrm{div})$, and thus the current in Fig. $6 \mathrm{~b}$ is measured by the other device for a short period time $(0.5 \mathrm{~s} /$ div $)$.

The voltage of BUS1 is higher than that of BUS2 due to the voltage drop in the SSBTS, and the voltage difference becomes higher when higher current flows via the SSBTS. After the interconnection, the three loads are sequentially connected
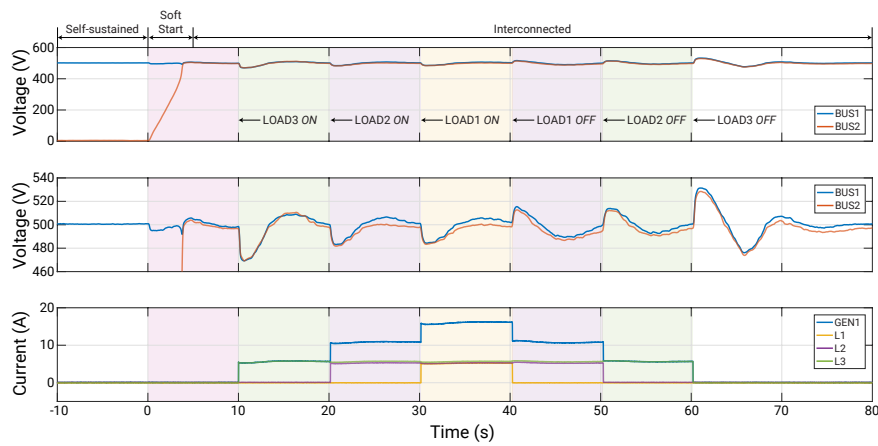

(a)

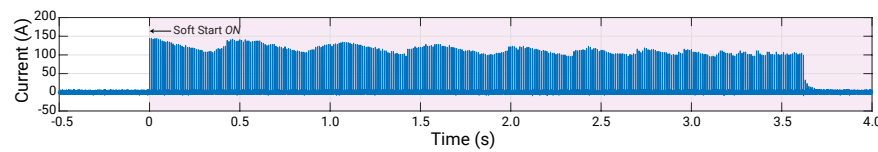

(b)

Fig. 6: Transient waveforms under GEN1 voltage control: (a) bus voltages and generator/load currents (10 s/div) and (b) current via the SSBTS during the soft start $(0.5 \mathrm{~s} / \mathrm{div})$.
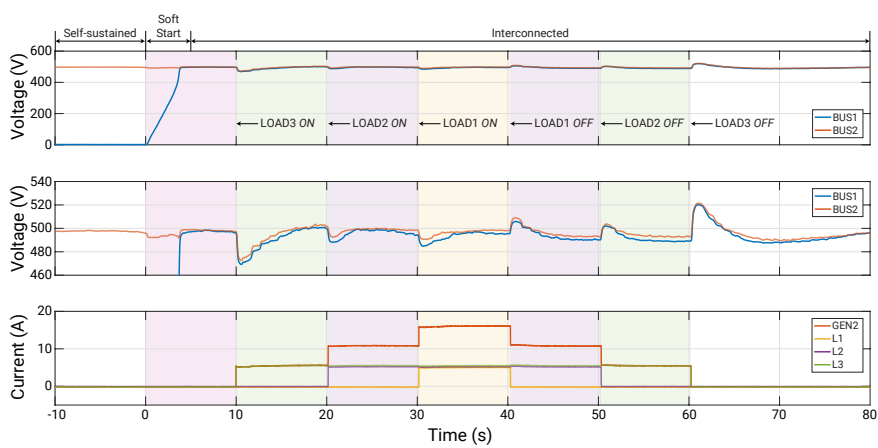

(a)

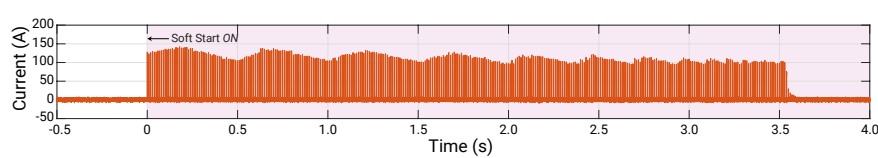

(b)

Fig. 7: Transient waveforms under GEN2 voltage control: (a) bus voltages and generator/load currents (10 s/div) and (b) current via the SSBTS during the soft start $(0.5 \mathrm{~s} /$ div $)$.

and disconnected with $10 \mathrm{~s}$ interval. The load changes bring with sudden voltage drops and rises, and the control system successfully regulates its bus voltage.

The soft start function and the DC voltage regulation implemented in GEN2 are also tested in Fig. 7. BUS2 is operated with its rated voltage and BUS1 is charged and interconnected. After the interconnection, the three loads are sequentially connected and disconnected, and the voltage control of GEN2 regulates the DC voltage of BUS2.

In this test, there is a voltage drop from BUS2 to BUS1 and it causes that the voltage of BUS2 is higher than that of BUS1. The other finding is that the voltage variations due to the load changes are less than those of the first test. This mainly comes from the different generator frequencies: GEN2 operated at approximately $200 \mathrm{~Hz}$ and GEN1 operated at $50 \mathrm{~Hz}$. GEN2 is operated with four times higher frequency compared to GEN1 and this increases the system reactance of GEN2. As a result, the increased reactance makes the GEN2 system less sensitive for the sudden load change.

\section{B. Power Sharing}

The two generators in the setup have to share loads in proportion to their power rating. Without the power sharing control, the two generators equally take a half of loading power if the two voltage levels are the same, while the power rating of GEN2 is 2.5 times higher than that of GEN1.

Generally, current- or power-based DC voltage droop control has been proposed and employed to provide the power sharing function in DC microgrids [17], [18]. In case of the current-based DC voltage droop control, source resistance is virtually added to generate the slope of droop characteristic. It is possible to control the load distribution between multiple generators by selecting different virtual resistances, i.e., higher virtual resistance should be considered for the generator with the lower power rating, and vice versa, as shown in Fig. 8. 


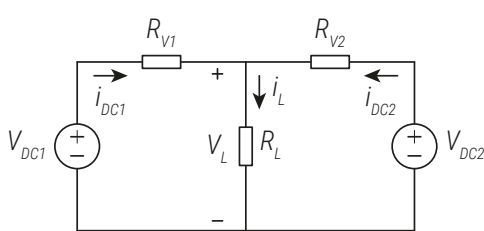

(a)

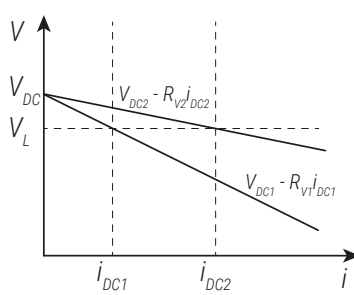

(b)
Fig. 8: General DC voltage droop control for two parallel voltage sources: (a) circuit diagram and (b) droop characteristics.

It is found that this general control scheme cannot be applied to the test setup that is the two-bus DC microgrids connected through the SSBTS (see Fig. 9). The first reason is related to the voltage drop $\left(V_{F}\right)$ in the SSBTS. The sign of the voltage drop is different depending on power flow due to its bidirectional feature and topology. For the power flow from $V_{D C 1}$ to $V_{D C 2}$,

$$
V_{D C 1}-R_{V 1} i_{D C 1}-V_{F}-R_{B U S} i_{12}=V_{D C 2}-R_{V 2} i_{D C 2}
$$

In case of the other flow,

$$
V_{D C 1}-R_{V 1} i_{D C 1}+V_{F}-R_{B U S} i_{12}=V_{D C 2}-R_{V 2} i_{D C 2}
$$

Because the conventional voltage droop control, as shown in Fig. 8, does not cover the voltage drop, its magnitude and sign have to be additionally considered.

The other reason originates from the voltage sensing resolution. If the resolution is not high, it is difficult to precisely regulate the power sharing or it is required to employ the steep droop slope. The former means poor power sharing function, and the latter can generate an issue in voltage regulation, e.g., a large variation of the reference voltage depending on load conditions. The voltage sensing issue is a more dominant cause why the test setup employs a new power sharing scheme. The DC voltage can be measured only with $1 \mathrm{~V}$ resolution in the setup, due to the combination of used sensors and an analogue/digital converter in the acquisition card of the PLC. After several tests, it became obvious that the $1 \mathrm{~V}$ resolution could not guarantee precise power sharing.

The employed power sharing scheme fixes the DC voltage of GEN2 with its DC voltage regulation and manipulates the AC voltage reference of GEN1 to supply electrical power within preselected ranges, as illustrated in Fig. 10. If GEN2 takes more power than the highest threshold, the AC voltage reference of GEN1 is increased with a preselected level $(\Delta V)$.

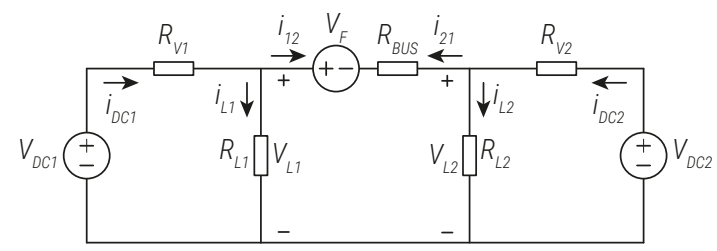

Fig. 9: Circuit diagram of two-bus DC microgrids connected through SSBTS.

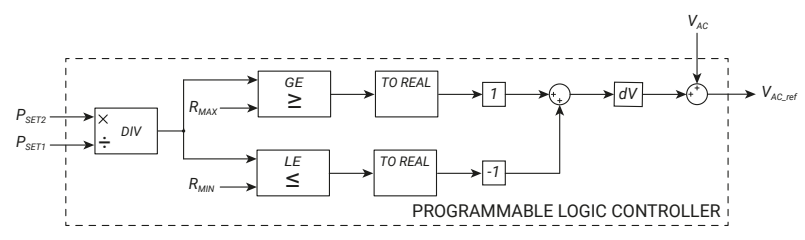

Fig. 10: Block diagram of power sharing control. The used parameters are: $R_{M A X}=3.3, R_{M I N}=1.9$, and $\Delta V=$ $0.05 \mathrm{~V}$.

For the other case, the AC reference is decreased at every control frequency of the PLC until the ratio is in the range.

The power sharing is demonstrated under the conditions of the two generators $O N$ and the initially closed SSBTS. Load1 (L1) installed at BUS1 is connected at $0 \mathrm{~s}$ and the two buses suffer voltage drops. The load is shared between the two generators during the transient period. When each bus is regulated at its rated voltage, GEN1 provides all the load current. It means that GEN2 has no contribution to the load due to the voltage drop in the SSBTS, while GEN2 $(25 \mathrm{~kW})$ has 2.5 time higher power rating than GEN1 $(10 \mathrm{~kW})$.

The power sharing function is activated at $40 \mathrm{~s}$. The function calculates the power sharing ratio between the two generators and gradually decreases the AC voltage reference of BUS1 in the tested case. Fig. 11a shows that power sharing is achieved

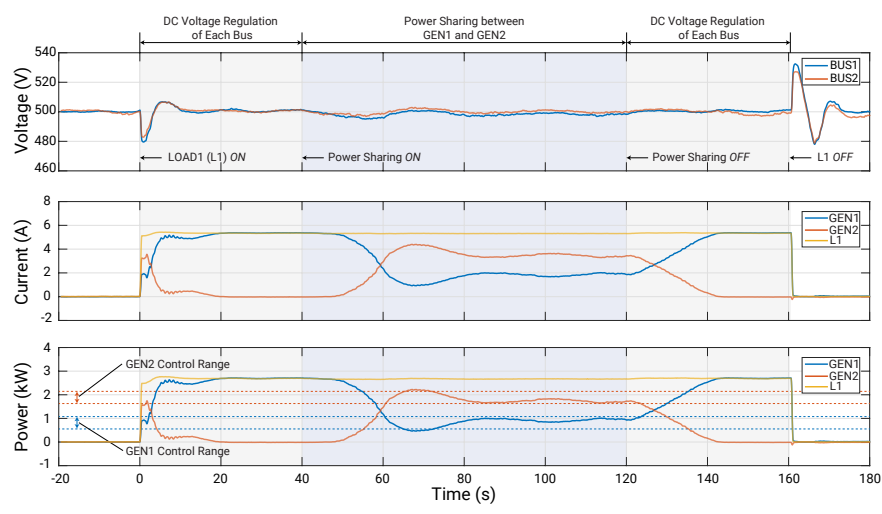

(a)
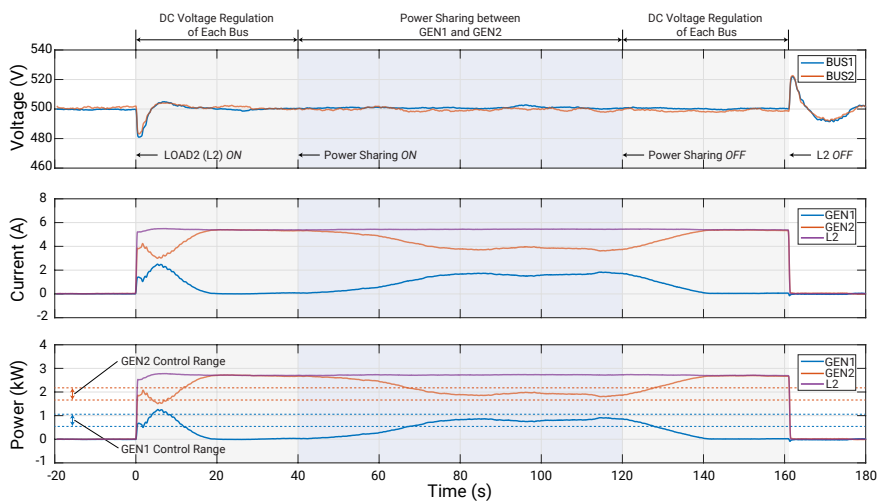

(b)

Fig. 11: Performance of power sharing function: (a) under the loading only at BUS1 and (b) under the loading only at BUS2. 
within each control range for $\mathrm{L} 1$. When the power sharing is deactivated, the voltage regulation control of BUS1 is restarted and GEN1 produces all the power for L1.

In the second case (see Fig. 11b), Load2 (L2) installed at BUS2 is connected at $0 \mathrm{~s}$. As expected, the load is powered only by GEN2 without the power sharing function. When the function is activated, the voltage of BUS1 is gradually increased to share the load within the control range.

The tests prove that the implemented function can control the power ratio between the two generators connected through the SSBTS that has different signs of the voltage drop depending on the current direction. Moreover, by accurately controlling the $\mathrm{AC}$ reference voltage, it is possible to overcome the aforementioned problem due to the low resolution in DC voltage sensing.

\section{Seamless Transition}

The used ultra-fast SSBTS allows for the seamless transition of system configuration without any extra costs and components. An interchange between open- and closed-bus operation can be achieved with a low system impact, as studied in the work of [19]. Furthermore, the power source can be transmitted promptly if one bus experiences a power outage during the open-bus operation (called seamless transition in the work). For the latter purpose, the seamless transition function is realized in the controller of the SSBTS.

Fig. 12 shows the diagram of seamless transition control implemented. The transition control can be activated during the open-bus operation. Once the ON signal (TRANSITION $O N$ in Fig. 12) becomes high, the $\mathrm{Q}$ output is reset low. Once the voltage difference between two buses is greater than a threshold value, the gate signal becomes and remains $O N$. If the current passing through the SSBTS reaches the fault detection condition (100 A in the work), the SSBTS is turned $O F F$. The used value of $V_{\text {threshold }}$ is $25 \mathrm{~V}$.

A seamless transition test is conducted under the conditions of the two generators $O N$ and the two loads at each bus (L1 and L2). Initially, the two buses are operated with the selfsustained mode (open-bus operation), and thus each generator supports the load at its bus. The voltage drop is commanded to BUS1 at $-2 \mathrm{~s}$ and the two buses are interconnected by the ultra-fast SSBTS when the voltage difference becomes the threshold value $(25 \mathrm{~V})$ at $0 \mathrm{~s}$ (see Fig. 13). Due to the loss of the source in BUS1, all load currents are supplied by GEN2 under the test condition.

For the other transition test, the SSBTS is turned $O F F$ at $8 \mathrm{~s}$ and the DC voltages of the two buses become the rated

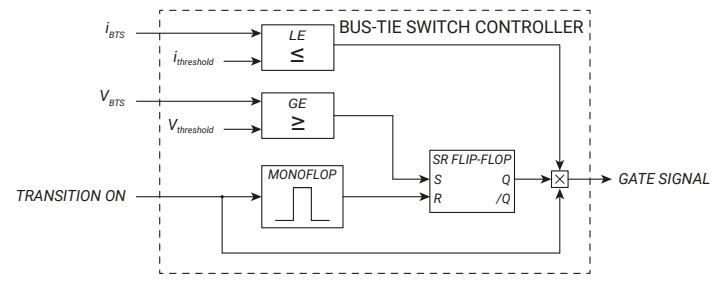

Fig. 12: Block diagram of seamless transition control.

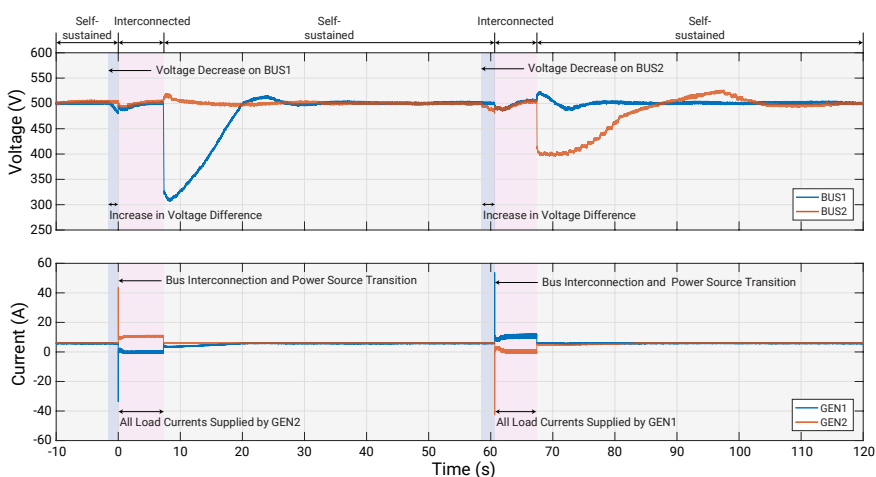

Fig. 13: Performance of seamless transition.

value. Same as the initial condition, each bus maintains its bus voltage and each load takes the power from each bus. The second transition is initiated by developing the voltage drop in BUS2 at $58 \mathrm{~s}$ and the interconnection occurs at $60 \mathrm{~s}$ with the voltage difference of $25 \mathrm{~V}$. The maximum transition current is approximately $55 \mathrm{~A}$ under the test conditions.

If the bus experienced the voltage drop has a permanent low-impedance fault, the transition current may reach the threshold value and the interconnection is not possible due to the protection action of the SSBTS.

The test result verifies that the seamless transition can be achieved with the SSBTS. It allows for operating the systems more flexible and reliable. Note that the seamless transition is demonstrated without relying on the supercapacitor banks integrated in the test setup.

\section{Demonstration OF EFFicient Systems with ESSS}

To verify superior features of the DC microgrids with ESSs, three system operating modes are experimentally demonstrated: load leveling, transient mitigation, and zero-emission.

\section{A. DC-DC Converter Control}

A bidirectional DC-DC converter is used (see Fig. 1), allowing for controlled charging and discharging of supercapacitors. Control is implemented in such a way that it is possible for energy storage system to take control of the DC bus voltage, in case that generator is out of function. The power setpoint of the converters can directly be set by the central controller or can be determined from the DC power droop characteristics. To enable load leveling, the direct power reference is fed to the converter controller. Conversely, the transient mitigation and zero-emission modes rely on the droop characteristics. The

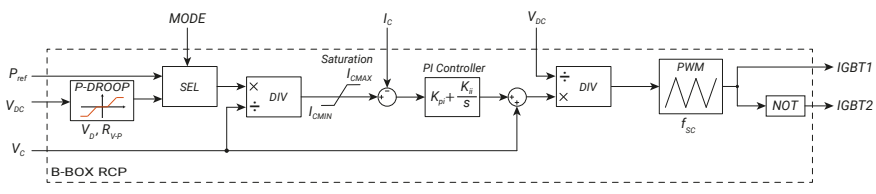

Fig. 14: Block diagram of DC-DC converter control [20], [21]. The used parameters are: $I_{C M A X}=8 \mathrm{~A}, I_{C M I N}=-8 \mathrm{~A}$, $K_{p i}=42.4, K_{i i}=3535.5$, and $f_{s c}=10 \mathrm{kHz}$. 
block diagram of the converter control is presented in Fig. 14. The diagram shows that the power is set by the closedloop control of the supercapacitor current $I_{C}$ in a conventional control structure. The setpoint current is determined from the setpoint power using the measured supercapacitor voltage $V_{C}$ and the value is saturated to protect both the converter and the supercapacitors. The switching signals are generated by PWM in a forced continuous operation. Despite lower conversion efficiency of this operation mode, this option is implemented for simplicity to ensure linearity of the controlled power for near zero setpoints.

\section{B. Load Leveling}

To maintain the generator load within optimal operating ranges, the difference between power demand and high/low threshold of the generator load should be compensated by ESSs. Thus, the central controller collects the total power demand and then sends the power reference to the ESSs . If there is no margin in the load leveling control, the actual generator load may not be in the control ranges because of the time delay caused by communication, ESS response, and so on. Including the margin $\left(P_{M A R G I N}\right)$, the block diagram of the load leveling control is presented in Fig. 15.

The load leveling control is demonstrated under the conditions of GEN1, L1, and L3 ON. One supercapacitor charges or discharges the energy to replicate highly variable loads from 4.1 to $7.1 \mathrm{~kW}$ within a minute. Once the power demand reaches $5.8 \mathrm{~kW}$ that is the upper threshold power to start the control ( $\left.P_{L L_{-} M A X}-P_{M A R G I N}\right)$, the other supercapacitor discharges its energy to take the power demand above the threshold. For lower power demand than $5.4 \mathrm{~kW}\left(P_{L L \_M I N}+\right.$ $\left.P_{M A R G I N}\right)$, the supercapacitor is operated with the charging mode to maintain generator loads within the load leveling range from 5.1 to $6.1 \mathrm{~kW}$ selected in the study.

The experimental test shows that the generator load (blue line) can be flattened within the control ranges while the power demand (blue-dotted line) is highly fluctuated. With the load leveling, it is possible to make the generator set to be operated at optimal operating ranges. Moreover, if the maximum power rating of the generator set is $6.5 \mathrm{~kW}$, two generator sets have to be run for high load conditions $(6.5-7.15 \mathrm{~kW}$ in the studied case). In this case, the power redundancy can be achieved by one running generator set with ESSs, as demonstrated. To sum up, DC microgrids equipped with ESSs can save large amounts of fuel for highly variable loads by operating the generator set

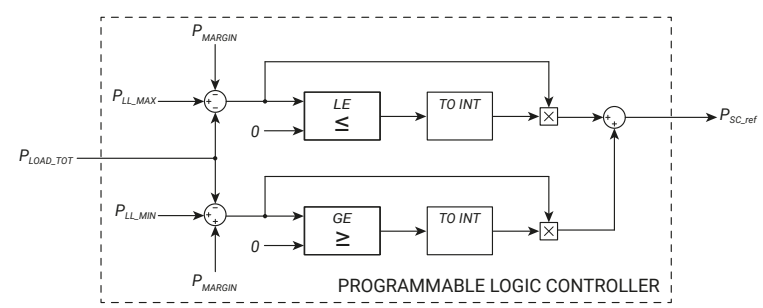

Fig. 15: Block diagram of load leveling control. The used parameters are: $P_{L L_{-} M A X}=6.1 \mathrm{~kW}, P_{L_{-} M I N}=5.1 \mathrm{~kW}$, and $P_{M A R G I N}=0 . \overline{3} \mathrm{~kW}$.

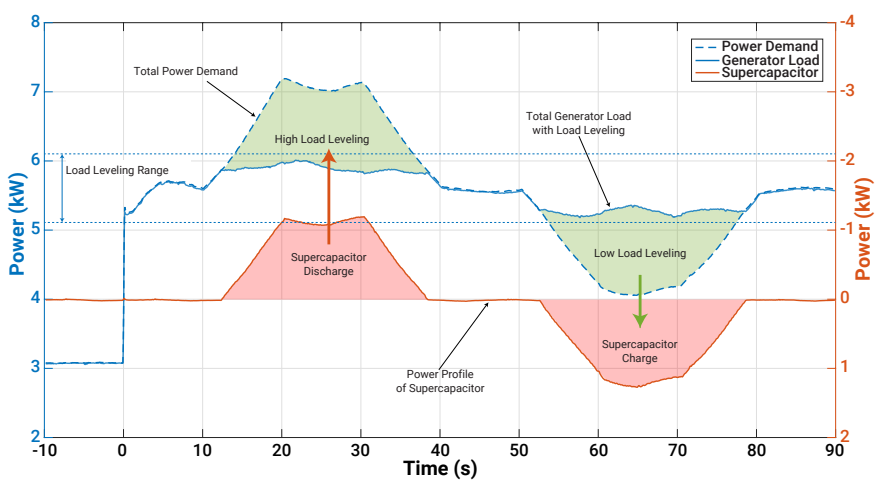

Fig. 16: Power profile of load leveling control.

in optimal operating ranges as well as by reducing the running generator set.

\section{Transient Mitigation}

Transient voltage is a representative indicator to recognize sudden load changes in DC microgrids, i.e., sudden load increases or decreases result in voltage drops or rises, respectively. Furthermore, higher load changes result in higher voltage changes. For these reasons, power droop control depicted in Fig. 17 is applied for the demonstration of transient mitigation.

In the test setup, the supercapacitor bank has a much faster response than the main power supply (synchronous generators combined with diode rectifiers). The transient performance of the DC microgrids is improved by using this fast acting device. When sudden load changes occur in the networks, those generate the voltage rises or drops. If the DC-link voltage becomes higher or lower than deadband voltages, the supercapacitor discharges or charges the energy according to the power droop control in Fig. 17.

Fig. 18a shows that the DC microgrids with ESSs have remarkably lower voltage drops and rises than those without ESSs. From the result, it could be stated that the system performance is greatly improved. It is also observed that the transients of the engine-generator set are mitigated with ESSs. While the power demand is changed in multi-step forms, the

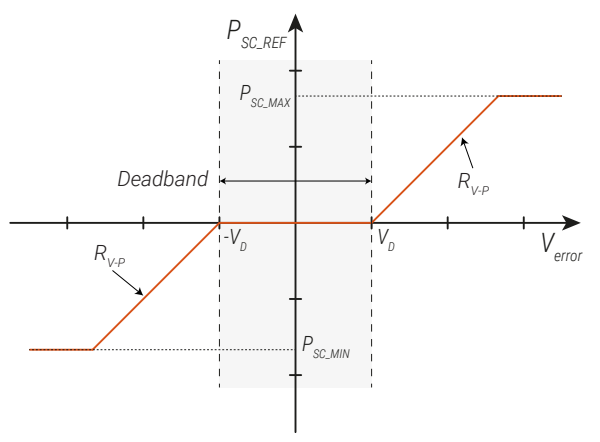

Fig. 17: Implemented power droop control. The used parameters are: $V_{D}=5 \mathrm{~V}, R_{V-P}=300 \mathrm{~W} / \mathrm{V}, P_{S C_{-} M A X}=3 \mathrm{~kW}$, and $P_{S C_{-} M I N}=-3 \mathrm{~kW}$. 


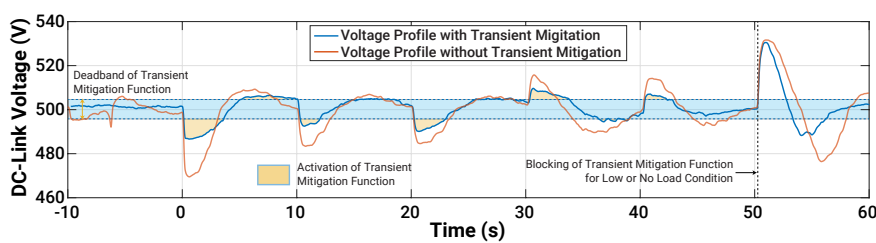

(a)

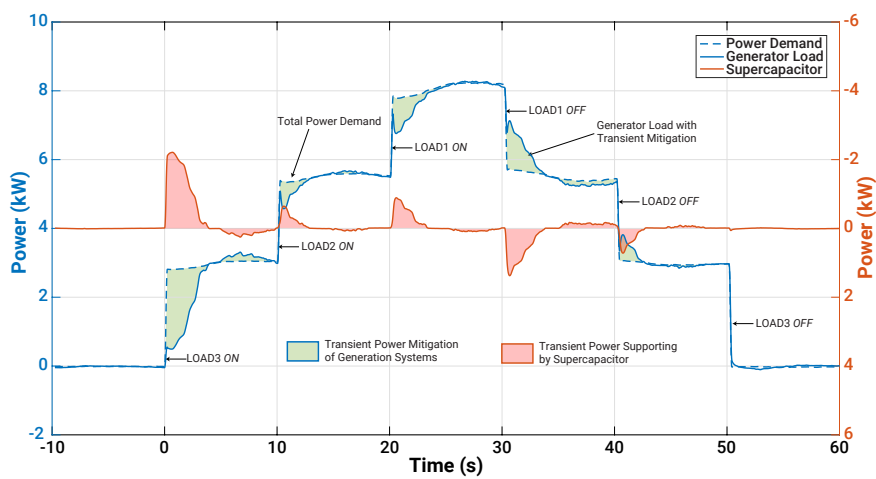

(b)

Fig. 18: Transient waveforms under transient mitigation control: (a) voltage profile and (b) power profile.

supercapacitor makes the actual generator load softer (see Fig. $18 \mathrm{~b})$. As the last point, the test proves that the regenerative energy can be stored in ESSs. To demonstrate the regenerative energy saving, two resistive loads are disconnected at $30 \mathrm{~s}$ and $40 \mathrm{~s}$, respectively. These sudden load decreases cause the voltage rises and these energies are stored in the supercapacitor with its power droop control. Note that the droop control to mitigate the transient is deactivated for low or no-load condition to avoid undesired voltage fluctuation.

\section{Zero-Emission}

Zero-emission operation is to avoid air pollutants from ships in a port to meet environmental regulations and to make a cleaner port. Hence, when a ship is approaching a port, the power source should be transited from onboard generators to ESSs. This transient is replicated by slowly turning $O F F$ the generators (GEN1 in the work) and then the energy storage systems (the supercapacitor banks) start to supply the power to the system with their power droop control, as shown in Fig. 19. In a port, grids (GEN2 in the work) can be connected to the ship through a shore-to-ship power supply. After this interconnection, by increasing the grid voltage slightly, the networks can be powered by the grids and this increased network voltage makes the ESSs to be charged according to their droop control. With the fully charged ESSs, the ship can leave the port with the zero-emission operating mode again.

The experimental test demonstrates that continuous power supply to onboard loads without air pollutant emission in a port can be realized with the power droop control of ESSs, which is newly applied for the zero-emission operating mode of marine DC microgrids.

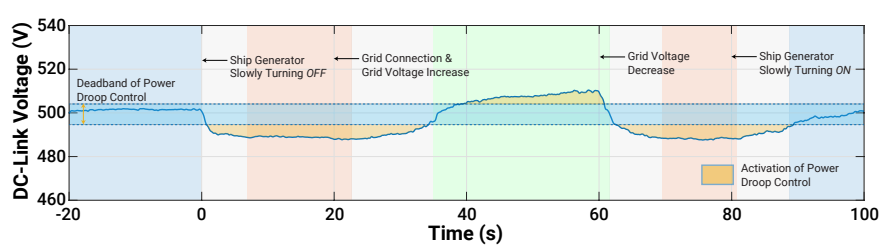

(a)

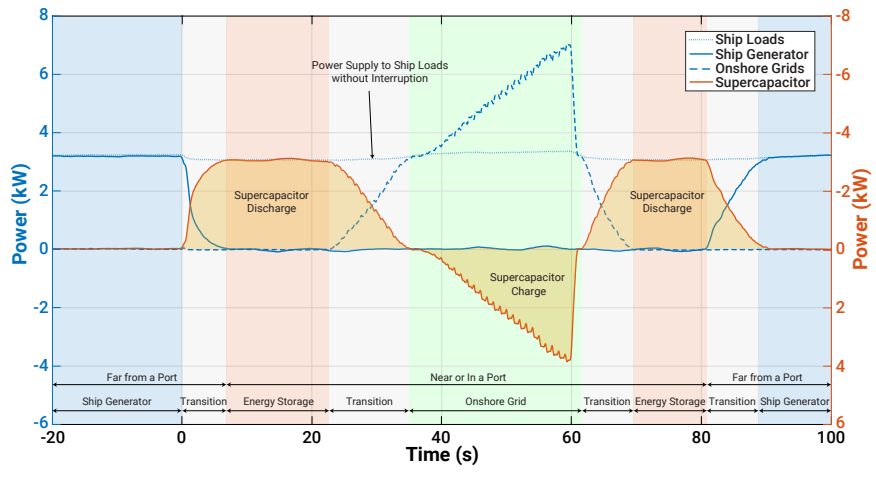

(b)

Fig. 19: Transient waveforms under zero-emission operating mode: (a) voltage profile and (b) power profile.

\section{CONCLUSION}

This paper has presented a wide range of DC microgrid operating modes, available with solid-state technologies and ESSs. The two-bus DC microgrids and the system controls are implemented to replicate marine DC microgrids.

The novel soft start and seamless transition functions of the SSBTS without additional circuits and components make marine DC microgrids more flexible and reliable as well as free from the voltage matching requirement during the synchronization. Integrating ESSs into marine DC microgrids allows for load leveling, transient mitigation, and zeroemission operating modes. With these modes, ship owners can save a large amount of fuel and avoid air pollutant emission in a port. From the above results, we conclude that marine DC microgrids with solid-state technologies and ESSs offer superior features in terms of the flexibility in energy management of the system, the fuel savings, and the environmental compliance in a port.

\section{REFERENCES}

[1] O. Alnes, S. Eriksen, and B. Vartdal, "Battery-powered ships: A class society perspective," IEEE Electrification Magazine, vol. 5, no. 3, pp. 10-21, Sep. 2017.

[2] S. O. Settemsdal, "Enhanced safety in power plant solutions proven by testing," ECPE Workshop, DC Grids, Technologies and Applications, Apr. 2018.

[3] J. F. Hansen, J. O. Lindtjørn, and K. Vanska, "Onboard DC Grid for enhanced DP operation in ships," in Dynamic Positioning Conference, Houston, 2011.

[4] B. Zahedi, L. E. Norum, and K. B. Ludvigsen, "Optimized efficiency of all-electric ships by dc hybrid power systems," Journal of Power Sources, vol. 255, pp. $341-354,2014$.

[5] "The step forward Onboard DC Grid," pp. 1-8, 2014.

[6] S. O. Settemsdal, E. Haugan, K. Aagesen, and B. Zahedi, "New Enhanced Safety Power Plant Solution for DP Vessels Operated in Closed Ring configuration," in Dynamic Positioning Conference, Houston, 2014. 
[7] E. Haugan, H. Rygg, A. Skjellnes, and L. Barstad, "Discrimination in offshore and marine dc distribution systems," in 2016 IEEE 17th Workshop on Control and Modeling for Power Electronics (COMPEL), pp. 1-7, Jun. 2016

[8] S. Kim, S. Kim, and D. Dujic, "Extending protection selectivity in dc shipboard power systems by means of additional bus capacitance," IEEE Transactions on Industrial Electronics, vol. 67, no. 5, pp. 3673-3683, 2020.

[9] G. Ulissi, S. Lee, and D. Dujic, "Scalable solid state bus tie switch for flexible shipboard power systems," IEEE Transactions on Power Electronics, pp. 1-1, 2020.

[10] A. Bindra, "Electric vehicle batteries eye solid-state technology - prototypes promise lower cost, faster charging, and greater safety," IEEE Power Electronics Magazine, vol. 7, no. 1, pp. 16-19, Mar. 2020.

[11] M. A. Hannan, M. M. Hoque, A. Hussain, Y. Yusof, and P. J. Ker, "State-of-the-art and energy management system of lithium-ion batteries in electric vehicle applications: Issues and recommendations," IEEE Access, vol. 6, pp. 19362-19378, 2018.

[12] S. Kim, G. Ulissi, S.-N. Kim, and D. Dujic, "Marine DC power distribution networks," in PCIM Europe 2019; International Exhibition and Conference for Power Electronics, Intelligent Motion, Renewable Energy and Energy Management, pp. 1-8, May. 2019.

[13] U. Javaid, F. D. Freijedo, D. Dujic, and W. van der Merwe, "Dynamic Assessment of Source-Load Interactions in Marine MVDC Distribution," IEEE Transactions on Industrial Electronics, vol. 64, DOI 10.1109/TIE.2017.2674597, no. 6, pp. 4372-4381, Jun. 2017.

[14] S. Kim, D. Dujic, and S.-N. Kim, "Protection schemes in low-voltage dc shipboard power systems," in PCIM Europe 2018; International Exhibition and Conference for Power Electronics, Intelligent Motion, Renewable Energy and Energy Management, pp. 1-7, Jun. 2018.

[15] G. Ulissi, S. Lee, and D. Dujic, "Solid state bus tie switch for shipboard power distribution networks," IEEE Transactions on Transportation Electrification, pp. 1-1, 2020.

[16] H. Saadat, Power System Analysis. McGraw-Hill, 2009.

[17] S. Peyghami, H. Mokhtari, P. C. Loh, P. Davari, and F. Blaabjerg, "Distributed primary and secondary power sharing in a droop-controlled lvdc microgrid with merged ac and dc characteristics," IEEE Transactions on Smart Grid, vol. 9, no. 3, pp. 2284-2294, 2018.

[18] C. Li, S. K. Chaudhary, M. Savaghebi, J. C. Vasquez, and J. M. Guerrero, "Power flow analysis for low-voltage ac and dc microgrids considering droop control and virtual impedance," IEEE Transactions on Smart Grid, vol. 8, no. 6, pp. 2754-2764, 2017.

[19] J. Xiao, P. Wang, and L. Setyawan, "Implementation of multiple-slackterminal dc microgrids for smooth transitions between grid-tied and islanded states," IEEE Transactions on Smart Grid, vol. 7, no. 1, pp 273-281, 2016

[20] A. Rufer, Energy Storage: Systems and Components. CRC Press, 2017.

[21] P. Grbovic, Power Conversion and Energy Storage Applications. John Wiley \& Sons, Ltd, 2013.

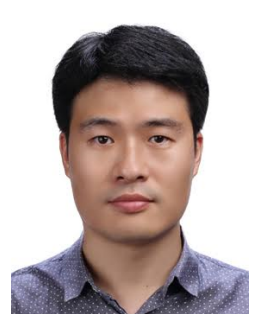

Seongil Kim (S'18-M'20) received the B.S. and M.S. degrees from Hanyang University, Ansan and Seoul, Republic of Korea, in 2005 and 2007, respectively, and the Ph.D. degree from École Polytechnique Fédérale de Lausanne (EPFL), Lausanne, Switzerland, in 2020, all in electrical engineering.

From 2007 to 2017, he was with Hyundai Heavy Industries Research Centre, Yongin, Republic of Korea, as a Senior Researcher working on high voltage and power system projects. From 2017 to 2020, he was with the Power Electronics Laboratory at EPFL as a Doctoral Assistant. He is currently with Hyundai Electric and Energy Systems Research Centre, Yongin, Republic of Korea, as a Senior Researcher working on power system and power electronics projects. He has authored or coauthored more than 40 scientific publications and has filed 14 patents. His current research interests include the areas of design, modeling, and protection of dc power systems.

Dr. Kim was the recipient of the IEEE Transportation Electrification Conference and Expo (ITEC) Student Paper Award in 2020.

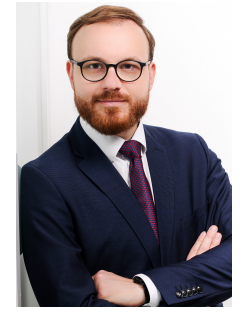

Jakub Kucka (S'14-M'19) received the bachelor and master degrees in electrical engineering from Czech Technical University, Prague, Czech Republic, in 2012 and 2014, respectively, and the Dr.Ing.(Ph.D.) degree from Leibniz University Hannover, Germany, in 2019. From 2014 to 2019, he was a Research Associate with the Institute for Drive Systems and Power Electronics, Leibniz University Hannover, Germany. Since 2020, he has been a Postdoctoral Researcher with the Power Electronics Laboratory, EPFL, Lausanne, Switzerland. He has authored more than 20 scientific publications, one tutorial, and filed three patent applications. His research interests include modular multilevel converters, converter control and design, and resonant converter topologies suitable for high-power dc applications.

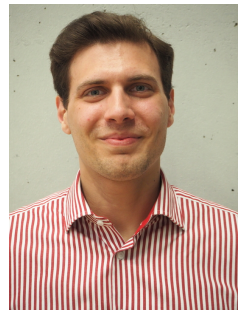

Gabriele Ulissi (S'18) received his B.Sc. degree in electrical engineering from the Polytechnic of Turin, Italy, in 2016 and M.Sc. degree in electrical engineering from the Ecole Polytechnique Fédérale de Lausanne (EPFL), Switzerland, in 2018. Since 2018, he is a Doctoral Assistant with the Power Electronics Laboratory at EPFL, Switzerland. His research focuses on semiconductor-based protection of dc systems.

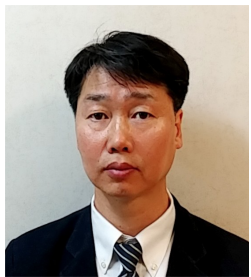

Soo-Nam Kim received the B.S., M.S., and Ph.D degrees in electrical engineering from Hanyang University, Ansan and Seoul, Republic of Korea, in 1996, 1998 and 2003, respectively.

From 2003 to 2017, he was a Head Researcher with Hyundai Heavy Industries Research Centre, Yongin, Republic of Korea, working on power system engineering for various areas. Since 2017, he has been a Senior Researcher with Hyundai Electric and Energy Systems Research Centre, Yongin, Republic of Korea, working on switchgear development for ships and smart grids. He is currently leading the Future Power Grid Research Department of Hyundai Electric and Energy Systems Research Centre. His current research interests include the areas of design and control of ecofriendly grids for ships, buildings and cities.

Dr. Kim was the recipient of the Minister's Award for Smart Grid, Republic of Korea, as a Meritorious Person, in 2012. 


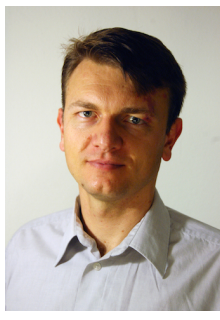

Drazen Dujic (S'03-M'09-SM'12) received the Dipl. -Ing. and M.Sc. degrees from the University of Novi Sad, Novi Sad, Serbia, in 2002 and 2005, respectively, and the Ph.D. degree from the Liverpool John Moores University, Liverpool, U.K., in 2008, all in electrical engineering.

From 2002 to 2006, he was with the Department of Electrical Engineering, University of Novi Sad as a Research Assistant, and from 2006 to 2009 with Liverpool John Moores University as a Research Associate. From 2009 to 2013, he was with ABB Corporate Research Centre, Switzerland, as a Principal Scientist working on the power electronics projects spanning the range from low-voltage/power switched-mode power supply (SMPS) in below kilowatt range to medium voltage high-power converters in a megawatt range. From 2013 to 2014, he was with ABB Medium Voltage Drives, Turgi, Switzerland, as a R\&D
Platform Manager, responsible for ABB's largest integrated gate-commutated thyristor (IGCT) based medium voltage drive ACS6000. He is currently with École Polytechnique Fédérale de Lausanne EPFL, Lausanne, Switzerland, as an Associate Professor in Electrical Engineering and the Director of the Power Electronics Laboratory. He has authored or coauthored more than 150 scientific publications and has filed 16 patents. His current research interests include the areas of design and control of advanced high-power electronics systems and high performance drives.

Dr. Dujic is an Associate Editor for the IEEE Transactions on Industrial Electronics, IEEE Transaction on Power Electronics, and IET Electric Power Applications. He is the recipient of the First Prize Paper Award by the Electric Machines Committee of the IEEE Industrial Electronics Society at IECON, in 2007. He was the recipient of the european power electronics and drives association (EPE) Outstanding Service Award in 2018, and the Isao Takahashi Power Electronics Award in 2014, for outstanding achievement in power electronics. 